\title{
El riesgo de cáncer de mama en pacientes con antecedente de patología mamaria benigna:
}

\author{
Eliana Maribel Quintero Roa
}

\section{RESUMEN}

Tomando en cuenta el impacto que sobre la vida de toda mujer lleva el riesgo de padecer Ca de manera, se realiza una revisión de la literatura, respecto de la influencia del diagnóstico histopatológico previo de lesiones no malignas, según la clasificación propuesta por los doctores Page y Dupont, y su posible malignización con el paso del tiempo.

Se realiza además un análisis más profundo de patologías clásicamente consideradas como "benignas" tales como los fibroadenomas y las cicatrices radiales mamarias, sus posibles variantes histopatológicas, y sus implicaciones como factores de riesgo para la paciente.

La más importante de la conclusiones, es la necesidad de implementar un seguimiento individualizado de cada paciente, según lo amerite la sumatoria de sus factores de riesgo, y la concientización de ella misma de estos riesgos, de manera tal que sea la misma paciente la más interesada en su propio control, para obtener así un diagnóstico temprano de patología mamaria maligna

PALABRAS CLAVES: Patología mamaria benigna, lesiones sin actividad proliferativa, lesiones con actividad proliferativa con y sin atipias, riesgo de Ca de mama.

\section{SUMMARY}

To asses the impact in the women life respect of the risk for breast cancer, this review examine the relation the between benign breast disease like Page and Dupont classification and the risk for breast cancer.

In this, you found an analysis for fibroadenomas and radial scars, usually consider benign breast tumors, its pathological variants, and if there are risk factors for breast cancer.

The most important conclusion, is the need to establish an individual pursuit to the patient, and the adequate explication to the risk factor, to obtain the collaboration for an early diagnosis of breast cancer.

KEY WORDS: Benign breast disease, non proliferative breast disease with and without atipias, breast cancer risk.

Las mujeres que han requerido una biopsia mamaria por alguna enfermedad benigna tienen un riesgo incrementado para $\mathrm{Ca}$ de mama, ya que pertenecen a un altamente seleccionado grupo de la población en quien se han identificado factores de riesgo para $\mathrm{Ca}$ de mama. Se ha intentado subdividir estas patologías homogéneamente, y realizar un pronóstico de las categorías relevantes respecto del riesgo de padecer $\mathrm{Ca}$ de mama. Estos esfuerzos sugieren que el riesgo de $\mathrm{Ca}$ de mama no es igual en todas las mujeres con antecedentes de enfermedad mamaria benigna. La variabilidad reportada en los diferentes estudios, respecto de los riesgos relativos en pacientes con este diagnóstico, posiblemente se deba a las diferencias de criterios usados para seleccionar las pacientes que requieren biopsia; las diferencias en la incidencia de otros factores de riesgo como la historia familiar, las diferentes prácticas quirúrgicas por parte de los cirujanos con respecto a la cirugía mamaria benigna, y la capacidad de seguimiento de estas pacientes (1).

A pesar de la controversia que existe sobre quién es la persona idónea para manejar la mama femenina, es el ginecoobstetra quien actúa como médico de atención primaria para la mujer, principalmente durante la edad fértil y los años perimenopáusicos, lo cual hace que el diagnóstico de enfermedades mamarias benignas, y $\mathrm{Ca}$ de mama en sus formas más curables sea detectado por este especialista (2).

\section{Enfermedades benignas de la mama:}

Clínicamente hablando, los síntomas mamarios benignos son frecuentes, calculándose que se presentan en aproximadamente el $50 \%$ de las mujeres. Evaluaciones 
post-mortem realizadas por Davis reportaron pruebas de enfermedad fibro-quística en el $58,5 \%$ de las autopsias realizadas, presentándose bilateralidad en el $43 \%$ de estas mujeres (2). La reunión de estas variantes histológicas, componen más de la mitad de todas las intervenciones quirúrgicas que se realizan sobre la mama femenina.

Alteraciones fisiológicas: La máxima incidencia de alteraciones mamarias benignas se presenta en mujeres de entre 30 y 50 años de edad, sugiriendo esto una posible etiología de disbalance hormonal como factor etiológico no confirmado de esta patología. Se trata de trastornos infrecuentes antes de la adolescencia, y que, muy ocasinalmente se desarrollan después de la menopausia.

\section{Enfermedad fibroquística}

Un diagnóstico patológico difuso, que aún hoy día se puede encontrar en la mayoría de los especímenes reportados, es "Enfermedad fibroquística"; que dice muy poco acerca de una variedad de lesiones que el espécimen de la biopsia puede contener.

Scanlon definió la enfermedad fibroquística desde el punto de vista clínico como "una condición en la cual se presentaban masas palpables bilaterales en la mama, usualmente asociadas con dolor e hipersensibilidad, que fluctuaban con el período menstrual y que disminuían progresivamente hasta la menopausia" (3).

Esta definición de mastalgia cíclica se aplica a la mayoría de mujeres en edad reproductiva, y al menos al 50\% de las mujeres que tienen mamas con irregularidades palpables, entonces, se plantea si es razonable definir como patológico un proceso que se presenta clínicamente en el $50 \%$ de las mujeres e histológicamente en el $90 \%$ (5).

Love y cols rebatieron el concepto de "enfermedad", y lo consideran como manifestaciones secundarias fisiológicas de las variaciones hormonales normales (4).

Para el desarrollo de este trastorno de múltiples patrones se considera que es básico el desequilibrio hormonal, principalmente el exceso absoluto de estrógenos o un déficit de progestágenos (4). Se sabe que el estradiol estimula las células epiteliales, y la progesterona induce el desarrollo de los acinos e interactúa con la acción mesenquimal de los estrógenos. La estimulación estrogénica persistente con variación cíclica de la secreción de progesterona parece ser la base de muchas distrofias mamarias benignas. Es por eso que se supone que el uso de anticonceptivos orales disminuye el riesgo de enfermedad fibroquística, dado que ofrece una fuente equilibrada de progestágenos y estrógenos (4). La combinación de las fluctuaciones hormonales y la respuesta tisular individual, determinan el grado de nodularidad y sensibilidad de la mama en cualquier momento (4).

La división entre estos cambios fisiológicos y la patología clínica depende no solo de la evaluación, sino también de la extensión, severidad y persistencia de estos hallazgos. La nodularidad fisiológica es clínicamente importante principalmente por la dificultad en distinguir un "nódulo dominante" que requiera biopsia por aspiración (4). Cuando a una mujer con una "condición fibroquística" se le realiza una biopsia mamaria, histológicamente se pueden encontrar un gran número de variantes, de las cuales es hoy evidente que las variables histológicas más importantes son el grado y carácter de hiperplasia y proliferación epitelial (5).

\section{Significado clínico de las alteraciones fibroquísticas}

Los patrones de patología mamaria incluidos en esta definición tienen importancia clínica por:

a) Clínicamente producen masas en la mama que deben ser diferenciadas del $\mathrm{Ca}$.

b) Frecuentemente en los reportes mamográficos se anota la presencia de microcalcificaciones.

c) Algunos pueden predisponer al desarrollo posterior de $\mathrm{Ca}$.

Page y Dupont (6) separaron las lesiones histológicas del tejido mamario benigno extirpado y las dividieron en solo 3 categorías de pronóstico oncológico.

\section{Lesiones sin actividad proliferativa}

\subsection{Tejido mamario normal.}

1.2 Fibroadenomas: tumor benigno más frecuente. Su relación con el Ca se ampliará más adelante.

1.3 Quistes: aproximadamente el $76 \%$ se presentan en la premenopausia, entre los 35-50 años. Pueden coexistir con adenosis y fibrosis, y algunos autores les asocian con secreción activa del epitelio apocrino bajo estímulo estrogénico.

1.4 Ectasia ductal: También llamada comedomastitis. Consiste en la dilatación de los conductos mamarios mayores e intermedios por debajo del pezón y areola. Se suele manifestar por la presencia de secreción de varios colores, pegajosa, bilateral y con afección de numerosos conductos. Se asocia a la presencia de dolor quemante, pruriginoso o sordo alrededor del pezón o de la areola, pudiéndose palpar inflamaciones tubulares tortuosas por debajo de ésta. Cuando se encuentra en fases más avanzadas puede desarrollarse la presencia de una masa que a veces recuerda un carcinoma mamario en estadio III (2). En algunos casos se acompaña de fibrosis e inflamación, que pueden producir retracciones, conformar masa o fistulizarse y presentar infección secundaria. Frecuentemente se encuentra en reportes mamográficos. Cuando hay recidivas frecuentes puede ser necesaria la resección de conductos terminales.

\subsection{Metaplasia apocrina}

1.6 Hiperplasia leve: Epitelio con profundidad superior a 2 células pero inferior a 4 .

\section{Enfermedades proliferativas}

\subsection{Enfermedadesproliferativas sin atipias}

2.1.1 Papiloma intraductal: Se manifiesta por una secreción serosa o sero-sanguinolenta por el pezón. Esta secreción suele ser espontánea y de un solo conducto, por lo cual usualmente es unilateral. La presión sobre un área de la areola provocará la secreción y por lo general, la lesión estará contenida en dicha área. Usualmente tienen un diámetro inferior a $1 \mathrm{~cm}$, generalmente 3-4mm. Ocasionalmente alcanzan tamaños de $3-5 \mathrm{cms}$. Macroscópicamente son tumores marrones o rosas que aparecen en conductos dilatados o quistes. Pueden presentar una 
configuración claramente papilar, o sésil. Microscópicamente está formado por múltiples ramificaciones y papilas anastomosantes, con un núcleo fibrovascular central en cada una de ellas y una capa de revestimiento de células epiteliales cuboideas o columnares (2).

\subsubsection{Hiperplasia moderada}

2.1.3 Hiperplasia ductal florida del tipo usual: El aumento en la cantidad de células alcanza 5 o más capas. Tiene tendencia a atravesar y distender los espacios donde se producen, asignándose la categoría de florida cuando las anormalidades son más pronunciadas, pero en verdad no existe un criterio estricto para asignarlas a un grupo u otro. Se encuentra en el $20 \%$ de las biopsias (7).

2.1.4 Cicatriz radial: Su descripción se ampliará más adelante.

2.1.5 Adenosis esclerosante: Se caracteriza por un aumento de la proliferación epitelial y de acinos, que con el tiempo pueden producir áreas de fibrosis conformando nodulaciones que disminuyen con la menopausia; se presenta característicamente en mujeres mayores de 30 años. Macroscópicamente a veces tienen una consistencia dura, cartilaginosa que comienza a aproximarse a la que se encuentra en el Ca de mama. Se discute si presenta un ligero aumento del riesgo de Ca de mama.

\subsection{Enfermedades proliferativas con atipias}

2.2.1 Hiperplasia ductal atípica: Las anomalías se localizan a nivel de los ductos mamarios, y comienzan a semejarse a los patrones observados en el carcinoma in situ sin llegar al diagnóstico inequívoco de éste (10), en cuanto a los rasgos cualitativos de patrones intercelulares y citológicos. La hiperplasia atípica se caracteriza por mostrar diversos grados de atipia celular y arquitectónica. Es la alteración asociada con la mayor posibilidad de $\mathrm{Ca}$ de mama.

2.2.2 Hiperplasia Lobular atípica: En esta variedad las anomalías celulares se localizan en los lóbulos mamarios.

Uno de los hallazgos más importantes de Page y Dupont fue que el $70 \%$ de las mujeres a quienes se les realizó el diagnóstico histológico de alguno de los componentes de la condición fibroquística no presentaron hallazgos que hicieran considerar un riesgo aumentado de padecer Ca de mama alguna vez en su vida (5).

Existen notables discusiones respecto de sí la paciente con una "condición fibroquística" tiene un mayor riesgo de desarrollar cáncer de mama. La mayoría de los estudios demuestran que no existe una mayor incidencia de este tipo de condición mamaria benigna en las mamas cancerosas que en las no cancerosas.

\section{Factores de riesgo para cáncer mamario}

Aunque se han identificado aproximadamente 20 factores de riesgo para padecer $\mathrm{Ca}$ de mama, solo el $21 \%$ de las pacientes afectadas tiene alguno. La importancia de identificarlos, radica en que estas pacientes requerirán un seguimiento más estricto y cuidadoso, que podría incluir la investigación de marcadores genéticos de riesgo para cáncer de mama (como el gen BRCA 1 del cromosoma 17q21 del cual se ha demostrado, su presencia incrementa en $80 \%$ el riesgo de la enfermedad), hasta la consideración de tratamientos profilácticos (8).

\section{Historia familiar de Cáncer (7)}

1.1 Madre con dx de cáncer mamario < de 60 años: aumenta el riesgo 2 veces.

1.2 Madre con dx de cáncer mamario > de 60 años: aumenta el riesgo 1.4 veces.

1.3 Historia de 2 parientes de primer grado con Ca de mama: aumenta el riesgo de 4 a 6 veces.

1.4 Pariente de primer grado con Ca bilateral < de 40 años: aumenta el riesgo 6 veces.

\section{Factores Hormonales (7)}

2.1 Menarquia temprana: <11 años y menopausia tardía >55 años: aumenta el riesgo 1.5 veces.

2.2 Uso de anticonceptivos orales: inicio de su uso a edades muy tempranas???.

2.3 Terapia de suplencia hormonal: No se ha podido demostrar que se aumente la incidencia de $\mathrm{Ca}$ de mama en las usuarias de TRH.

\section{Otros factores (7)}

Desproporción de talla-peso, obesidad, licor, tabaquismo, dietas ricas en grasas de origen animal, primer parto después de los 30 años, no haber lactado: aumentan el riesgo 1.5 veces.

\section{Factores de riesgo para cáncer de mama en mujeres con enfermedad mamaria proliferativa}

Las mujeres que tienen un antecedente de biopsia mamaria por una enfermedad benigna tienen un riesgo incrementado de diagnóstico de $\mathrm{Ca}$ de mama.

Los estudios retrospectivos realizados se basan en los hallazgos histopatológicos de las biopsias ya tomadas, lo cual incluye un sesgo, al demostrarse que una mujer ya sometida a una biopsia por patología mamaria benigna está más propensa a que se le realice una segunda biopsia, quizá debido a una mayor vigilancia o incluso a la deformación que provoca la cirugía previa. Estas biopsias reflejan un espectro de condiciones histológicas que varían desde el tejido mamario normal, hasta el $\mathrm{Ca}$ in situ. Los trabajos realizados para clasificar el riesgo secundario dependiendo del diagnóstico histológico no muestran resultados uniformes (8).

El riesgo de padecer $\mathrm{Ca}$ de mama en las pacientes con diagnóstico de enfermedad no proliferativa es el mismo de las pacientes de su misma edad que no padecen ninguna alteración (RR 1) (9).

Las pacientes con diagnóstico de enfermedad proliferativa sin atipias tuvieron un RR de 1.6, 1.8, 1.3, 1.9 (cuadro 1) veces el riesgo de padecerCa de mama respecto de las mujeres sin patología mamaria $(9-10,12,14)$.

Sólo e12\% de las mujeres con lesiones no proliferativas y $4 \%$ de las mujeres con lesiones proliferativas sin atipias que no tienen ningún otro antecedente positivo para $\mathrm{Ca}$ de mama, desarrollarán Ca de mama en los siguientes 15 años (10).

De las pacientes con diagnóstico de trastorno proliferativo con atipias sin antecedentes familiares:

$\checkmark$ El trabajo de London y Conolly basado en la cohorte prospectiva del estudio de las enfermeras, con la 
evaluación de 121 casos y 488 controles reportó un RR de 3.7 con un IC 95\% de 2.1-6.8.

$\checkmark \quad$ De la evaluación realizada por Dupont y cols en 1993, se reporta un RR de 4.3 con un IC 95\% 1.7-11.

$\checkmark$ Del trabajo de Dupont y Page publicado en NEJM en 1985, se relata un RR de 5.3 con un IC 95\% 3-1-8.8.

McDivitt y cols informan un RR de 2.6 con un IC $95 \%$ de 1.6-4.1 (Ver cuadro 1).

Las mujeres con hiperplasia atípica pero sin antecedentes familiares de importancia presentaron una elevación de 4-5 veces en el riesgo de padecer $\mathrm{Ca}$ de mama respecto de las pacientes sin enfermedad proliferativa. Ahora, las pacientes con diagnóstico de enfermedad proliferativa con atipias, y antecedente familiar de riesgo

Cuadro 1

RIESGO RELATIVO DE PADECER CA DE MAMA SEGÚN LA CLASIFICACIÓN DE PAGE Y DUPONT EN LAS DIFERENTES PUBLICACIONES

\begin{tabular}{|c|c|c|c|}
\hline ESTUDIO & $\begin{array}{c}\text { ENF. NO } \\
\text { PROLIFERATI } \\
\text { VA RR IC } 95 \%\end{array}$ & $\begin{array}{c}\text { ENF. } \\
\text { PROLIFERATIVA } \\
\text { SIN ATIPIAS RR } \\
\text { IC } 95 \%\end{array}$ & $\begin{array}{c}\text { ENF. } \\
\text { PROLIFERATIVA } \\
\text { CON ATIPIAS RR IC } \\
95 \%\end{array}$ \\
\hline $\begin{array}{l}\text { LONDON Y } \\
\text { CONOLLY }\end{array}$ & & $1.6(1-2.5)$ & $3.7(2.1-6.8)$ \\
\hline $\begin{array}{l}\text { McDIVITT Y } \\
\text { COLS }\end{array}$ & $1.5(1.3-1.9)$ & $1.8(1.3-2.4)$ & $2.6(1.6-4.1)$ \\
\hline $\begin{array}{c}\text { DUPONT Y } \\
\text { FRITZ }\end{array}$ & 1 & $1.3(0.77-2.2)$ & $4.3(1.7-11)$ \\
\hline $\begin{array}{c}\text { DUPONT Y } \\
\text { PAGE }\end{array}$ & & $1.9(1.2-2.9)$ & $5.3(3.1-8.8)$ \\
\hline
\end{tabular}

para Ca de mama, tuvieron un incremento de 5 veces más sobre el riesgo basal ya anotado (9-12). (Ver cuadro 2).

El riesgo de Ca de mama entre las mujeres con diagnóstico de hiperplasia atípica fue mayor entre las premenopáusicas versus las postmenopáusicas, lo cual es consistente con la aguda caída en el porcentaje de incremento en el $\mathrm{Ca}$ de mama en las pacientes post-menopáusicas, una observación probablemente relacionada con la influencia de la disminución hormonal del período perimenopáusico y el Ca de mama. (Ver cuadro 2).

Es lógico que el ciclo de producción de relativamente grandes cantidades de estradiol y progesterona pueden
Cuadro 2

RIESGO DE CANCER DE MAMA ASOCIADO CON ENFERMEDAD PROLIFERATIVA DE LA MAMA E HIPERPLASIA ATIPICA CANCER. 71;4:1258-1265

\begin{tabular}{|c|c|c|c|}
\hline FACTOR DE RIESGO & RR & $95 \%$ IC & VALOR P \\
\hline $\begin{array}{c}\text { NO ENFERMEDAD } \\
\text { PROLIFERATIVA }\end{array}$ & 1 & & \\
\hline $\begin{array}{c}\text { ENFERMEDAD PROLIFERATIVA } \\
\text { SIN ATIPIA }\end{array}$ & 1.3 & $0.77-2.2$ & 0.32 \\
\hline HIPERPLASIA ATIPICA & 4.3 & $1.7-11$ & 0.002 \\
\hline HISTORIA FAMILIAR & 2.4 & & \\
\hline $\begin{array}{c}\text { ENFERMEDAD PROLIFERATIVA } \\
\text { SIN ATIPIA + HISTORIA FAMILIAR }\end{array}$ & 2.6 & $1.0-6.4$ & 0.05 \\
\hline HIPERPLASIA ATIPICA + & 22 & $2.4-203$ & 0.006 \\
HISTORIA FAMILIAR & & & \\
\hline HIPERPLASIA ATIPICA \\
PREMENOPAUSICA & 12 & $2.0-68$ & 0.006 \\
\hline HIPERPLASIA ATIPICA \\
POSTMENOPAUSICA & 3.3 & $1.1-10$ & 0.04 \\
\hline
\end{tabular}

afectar las lesiones proliferativas así como el tejido mamario normal (12).

Riesgos individuales de padecer Ca de mama según el diagnóstico histopatológico

\section{Cicatrices radiales}

Su importancia radica en que tanto en las exploraciones macro y microscópicas pueden parecer carcinomas. Habitualmente miden menos de $1 \mathrm{~cm}$ y microscópicamente son irregulares, en forma de estrella, con un núcleo fibroso central que contiene elementos glandulares atrapados (1), de color gris-blanquecino, e induradas con retracción central con aspecto muy similar al carcinoma. El tejido mamario adyacente tiene diversos grados de hiperplasia intraductal y adenosis (1). Se caracteriza porque la proliferación epitelial aumenta y con el tiempo puede producir áreas de fibrosis conformando nodulaciones que disminuyen en la menopausia. Se presenta en mujeres mayores de 30 años (7). Son un tipo específico de lesión mamaria benigna, caracterizada microscópicamente por tener un núcleo fibroelástico con ductos y lóbulos radiados. Estos ductos y lóbulos muestran varias alteraciones incluyendo quistes y lesiones proliferativas. Usualmente se trata de hallazgos incidentales en tejido mamario removido por otras causas. La similitud morfológica de estas cicatrices con el $\mathrm{Ca}$, y el hallazgo de $\mathrm{Ca}$ en algunas cicatrices ha sugerido que estas lesiones pueden representar una fase temprana en el desarrollo de ciertos tipos de $\mathrm{Ca}$ de mama. Según el estudio de Jacobs y cols, se concluyó que el riesgo de $\mathrm{Ca}$ 
de mama fue mayor entre las mujeres con cicatrices grandes $(>4 \mathrm{~mm})$ y que se presentan en mayor número.

Igualmente no se encontró relación con mayor riesgo de presentar la enfermedad en la mama contralateral (13).

\section{Fibroadenomas}

Son los tumores benignos más frecuentes de la mama. Están compuestos por tejido epitelial y estromal. Se pueden presentar a cualquier edad, pero son más comúnmente diagnosticados alrededor de los 20 años, cuando el $\mathrm{Ca}$ de mama es extremadamente raro; es por esto que los fibroadenomas tradicionalmente no se han relacionado con el riesgo de $\mathrm{Ca}(7)$. Se considera que solo el componente fibroso (estromal) es clonal. Parece surgir del estroma intralobulillar especializado, lo cual podría explicar porqué estas lesiones no aparecen en otras localizaciones de las partes blandas.

Los fibroadenomas crecen como nódulos esféricos generalmente netamente delimitados y se mueven libremente respecto ala sustanciamamaria de alrededor. Usualmente aparecen en el cuadrante super-externo de la mama. Varían de tamaño desde menos de $1 \mathrm{~cm}$ hasta formas gigantes de 10$15 \mathrm{cms}$. Los fibroadenomas exhiben un amplio rango de patrones citológicos e histológicos.

\section{CLASIFICACION (8):}

\section{Simples}

2. Complejos: El componente epitelial de los fibro adenomas se clasifica como complejo si contiene:

$\checkmark$ - Quistes mayores de 3mm de diámetro.

$\checkmark$ - Adenosis esclerosante.

$\checkmark$ - Calcificaciones epiteliales.

$\checkmark$ - Cambios papilares apocrinos.

Cuando se anexa suficiente parenquima adyacente al fibroadenoma, este es clasificado respecto de la clasificación de Page de enfermedad mamaria benigna.

\section{Cuadro 3}

RIESGO DE CA MAMARIO INVASIVO EN PACIENTES CON FIBROADENOMAS 8

\begin{tabular}{|c|c|c|}
\hline FACTOR DE RIESGO & RR & IC 95\% \\
\hline PRESENCIA DE FIBROADENOMA & 1.6 & $1.3-2$ \\
\hline NO COMPLEJO & 1.42 & $1.1-1.8$ \\
\hline COMPLEJO & 2.24 & $1.6-3.2$ \\
\hline $\begin{array}{c}\text { PARENQUIMA NO EVALUADO } \\
\text { PIBROADENOMA SIN ENFERMEDAD }\end{array}$ & 1.59 & $1.0-2.5$ \\
\hline $\begin{array}{c}\text { FIBROADENOMA CON ENFERMEDAD } \\
\text { PROLIFERATIVA SIN ATIPIA }\end{array}$ & 1.48 & $1.1-1.9$ \\
\hline FIBROADENOMA + HIPERPLASIA \\
ATIPICA
\end{tabular}

De las 1.835 pacientes evaluadas por Page y Dupont en su estudio, concluyeron que de estas, dos terceras partes no presentaban fibroadenomas complejos, y no existía evidencia que sugiriera un mayor riesgo de $\mathrm{Ca}$ de mama en estas pacientes, si no se asociaba a la presencia de antecedentes familiares positivos (RR 1.08 IC $0.79-1.49$.) (Ver cuadro 3)

Comparativamente, las pacientes con diagnóstico de fibroadenomas complejos tuvieron una significancia mayor de peso estadístico como factor de riesgo para padecer $\mathrm{Ca}$ de mama. ( $\mathrm{P}=0.006$. $)$. Además cuando lo anterior, se asoció a la presencia de parenquíma adyacente afectado por una enfermedad proliferativa y peor aún hiperplasia atípica, se documentó un RR de 4.77 con un IC de $1.5-158$, (Ver cuadro 3).

$\mathrm{Si}$ además asociamos la presencia de antecedentes familiares positivos a todo lo anteriormente anotado, se asocia con una incidencia del $20 \%$ de $\mathrm{Ca}$ de mama durante los siguientes 25 años después del diagnóstico.

En el estudio de Page y Dupont, se encontró una mayor incidencia de enfermedad proliferativa alrededor de los fibroadenomas complejos ( $\mathrm{p}=0.002$ ), por lo cual se recomienda en lo posible incluir algo de tejido adyacente al fibroadenoma si técnicamente es posible hacerlo 8 .

En esencia, el diagnóstico de un fibroadenoma complejo, asociado a un antecedente familiar positivo, debe servir como incentivo para recomendar una evaluación mamográfica estricta, que inicie a partir de los 35 años.

De otra parte McDivitt y cols reportan un OR de 1.7 IC (1.1-2.5) para padecer $\mathrm{Ca}$ de mama en las pacientes con antecedentes de fibroadenomas.

De cualquier manera, algunos autores especifican que la mayoría de los fibroadenomas diagnosticados, no son tratados quirúrgicamente, y la decisión de una excisión quirúrgica, puede ser influenciada por la presencia de factores de riesgo individuales, que pueden actuar como factores de confusión, y sesgar los resultados obtenidos, basándose solo en los reportes patológicos de las muestras resecadas.

\section{Conclusiones}

Análisis del riesgo en pacientes que han padecido alguna enfermedad benigna mamaria alguna vez en su vida, de padecer posteriormente cáncer de mama muestran que:

* Para las mujeres que tienen una biopsia previa compatible con una enfermedad mamaria benigna, el riesgo general de diagnóstico de cáncer de mama es de 1.7 veces más que aquellas pacientes que no tienen historia de cirugía por enfermedad mamaria benigna. (RR 1.7 IC 95\% 1.5-2.0), esto explicado posiblemente por un seguimiento más estricto por parte de la paciente, y de su médico sumado a los cambios pronósticos dados por el hallazgo histopatológico individual.

* El diagnóstico histopatológico de enfermedad mamaria benigna proliferativa indica un aumento en el riesgo de padecer $\mathrm{Ca}$ de mama, pero este riesgo es mucho mayor si el diagnóstico es de hiperplasia atípica mamaria13.

* Se ha encontrado que la presencia de un fibroadenoma simple, solo aporta el aumento de riesgo de 
padecer $\mathrm{Ca}$ de mama que le confiere el convertirse en un antecedente quirúrgico mamario benigno, y la presencia de fibroadenomas complejos aumentan aún más el riesgo de $\mathrm{Ca}$ de mama si se asocian a presencia de hiperplasia y/o atipias (13).

* Se recomienda en múltiples estudios evitar el término inespecífico de "enfermedad fibroquística" en los reportes patológicos, ya que eliminan la posibilidad de una adecuada interpretación por parte del clínico, respecto de los riesgos ya enumerados de asociarse con $\mathrm{Ca}$ de mama.
* Está por determinar pautas de seguimiento claras para las pacientes en quienes se realizan los diagnósticos de las enfermedades mamarias benignas, con respecto a su posibilidad de desarrollar $\mathrm{Ca}$ de mama en un futuro.

* Tal vez lo más importante hoy día es aclarar a la paciente sobre su real porcentaje estadístico de patología maligna futura, para así proceder a una detección oportuna, sin dar información alarmista, pero tampoco menospreciando los hallazgos anotados, más aún en pacientes con antecedentes familiares de riesgo.

\section{BIBLIOGRAFIA}

1. Bonardi C, Zappa M, Giorgi D. Risk of breast cancer subsequent to histological or clinical diagnosis of fibroadenoma-retrospective longitudinal study of 3938 cases. Annals Oncology. 1997; 8: 297-300.

2. DiSaia, P, Creasrnan W. Oncología Ginecológica Clínica. Quinta Edición. 1999; 387-433.

3. Scanlon E.F. The early diagnosis of breast Cancer. Cancer 1981; 48: 523 6.

4. Love S, Gelman R. Fibrocystic "Disease" of the breast - a nondisease? The new England Journal of Medicine. 1982; 307: 1010-1014.

5. Hutter R. Goodbye to "Fibrocystic Disease". The New England Journal of Medicine. 1985; 312(3): 179-181

6. Page DL, Vanderzagg R, Roger LW, Williams LT. Relation between component parts of fibrocystic disease complex and breast cancer. JNCI. 1978; 61: 1055-63.

7. Guias de Manejo de Tumores Mamarios. Revista Colombiana de Ginecología y Obstetricia. Suplemento 48(4): 9-24.

8. Dupont W, Page D. Fritz F, et al. Long-Term Risk of Breast Cancer in women with fibroadenoma. The New England Journal of Medicine. 1994; 331: 10-15.
9. Dupont W, Fritz F, William H, et al. Breast Cancer risk associated with proliferative breast disease and Atypical hyperplasia. Cancer. 1993; 71: 1258-1265.

10. Dupont W, Page D. Risk factors for breast cancer in women with proliferative breast disease. The new England Journal of Medicine.1985; 312: 146-151.

11. Roberts M, Jones V, Elton R, Fortt R, et al. Risk of breast cancer in women with history of bening disease of the breast. British Medical Journal. 1984; 288: 275-278.

12. London S, Cono11y J, Schnitt S. A prospective Study of benign breast disease and the risk of breast cancer. JAMA. 1992; 267: 941-944.

13. Jacobs T, Byrne C, Colditz G, Conolly J, Schnitt S. Radial Scars in benign breast-biopsy specimens and the risk of breast cancer. The new England Journal of Medicine. 1999; 340: 430-435.

14. McDivitt R, Stevens J, Lee N, Wingo p, et al. Histologic Types of Benign breast disease and the risk for breast Cancer. Cancer. 1992; 69: 14081414.

15. Cook, MG. Bening breast disease: the relationship between its histologycal features and risk factors for breast cancer. Pathology. 1991; 23(4): $286-90$. 